\title{
Allzeit bereit?
}

ARBEITSZEITRECHT Neue Arbeitszeitmodelle in der Pflege kommen derzeit immer mehr in Mode. Klarheit für Arbeitgeber und Arbeitnehmer hat das aber leider bisher nicht zur Folge. Rufbereitschaft, Bereitschaftsdienst und neuerdings sogenannte „Stand-by-Dienste“ oder auch „Flexi-Modelle“ sorgen mehr denn je für Verwirrung. Aufgrund zahlreicher Leserzuschriften widmen wir uns deshalb wiederholt der Frage: Wer darf was wann anordnen und wie ist es letztlich zu vergüten? Unsere Fachanwälte klären über die aktuelle Rechtslage auf.

Daniel Balzert, Dr. Tobias Weimer

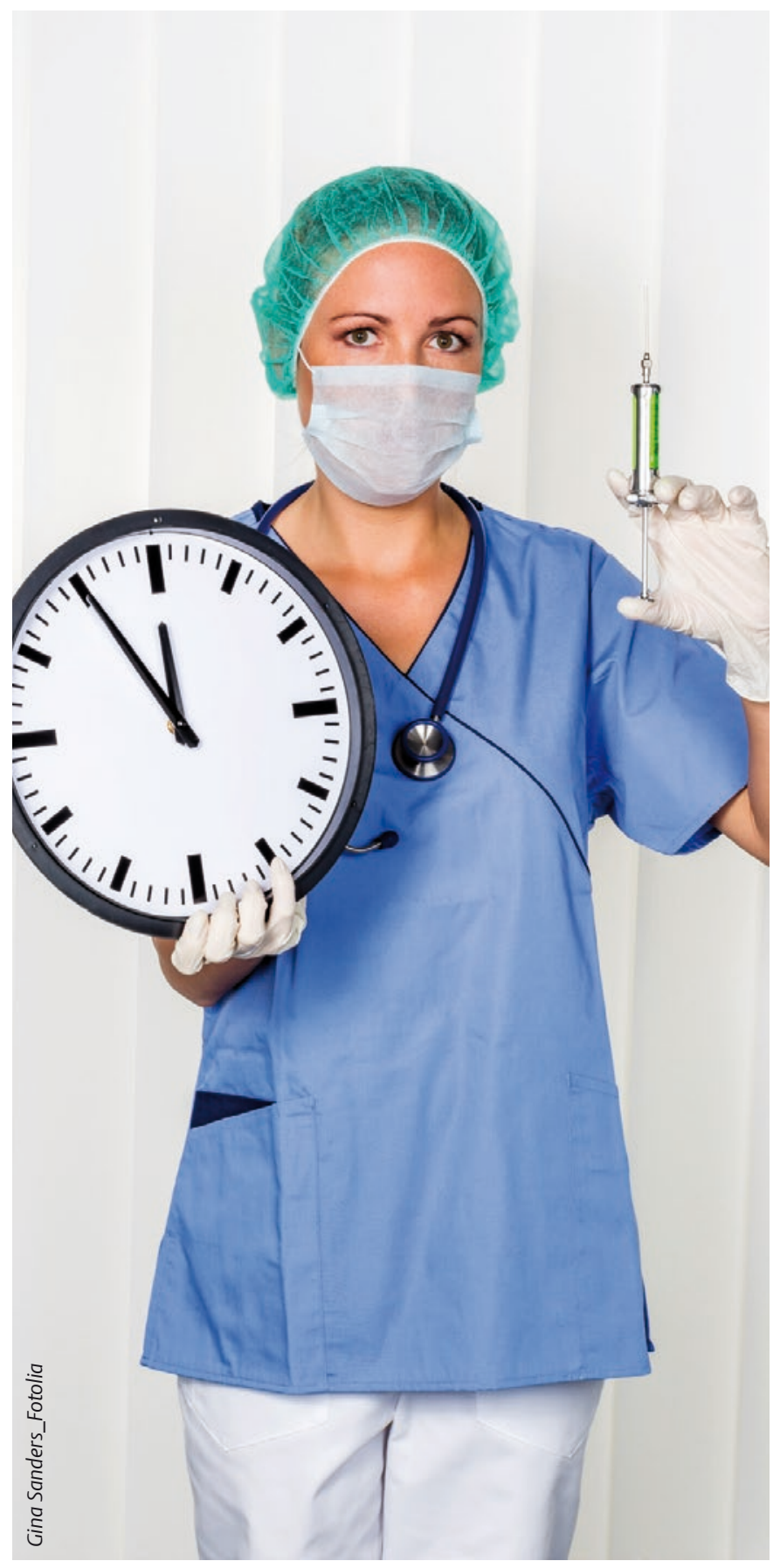

\section{Der Grundsatz: das Arbeitszeitgesetz}

Ausgangspunkt für eine juristische Beurteilung sind zunächst die gesetzlichen Bestimmungen des Arbeitszeitgesetzes (ArbZG).

Denn welche Regelungen in einem Arbeitsvertrag zwischen dem Arbeitgeber und dem einzelnen Arbeitnehmer grundsätzlich zulässig und was die absoluten Grenzen des Erlaubten in puncto Arbeitszeit, Mehrarbeit und Überstunden sind, ist darin festgehalten.

Geregelt ist dort beispielsweise eine werktägliche Höchstarbeitszeit von acht Stunden, das ist das maximale Pensum, das von Montag bis Samstag pro Tag gearbeitet werden darf. Dieser Zeitraum kann um zwei weitere Stunden pro Werktag verlängert werden, wenn innerhalb von sechs Kalendermonaten oder innerhalb von 24 Wochen der Durchschnitt von acht Stunden pro Werktag nicht überschritten wird ( $§ 3$ ArbZG).

Auch bestimmt das Gesetz, dass nach jedem Arbeitstag eine sogenannte „Ruhezeit“ von mindestens elf Stunden einzuhalten ist, also zwischen Arbeitsende und neuem Arbeitsbeginn fast ein halber Tag nichts gemacht werden darf ( $§ 5$ ArbZG).

Schließlich formuliert das Arbeitszeitgesetz, was überhaupt unter Arbeitszeit, Bereitschafts- und Nachtdienst oder Arbeitsbereitschaft zu verstehen ist. Es werden jedoch keine Regelungen darüber getroffen, ob oder wie die geleisteten Arbeitsstunden zu vergüten sind. Das Arbeitszeitgesetz ist vollständig „vergütungsneutral“.

\section{Abweichende betriebliche Regelungen}

Darüber hinaus kann in Unternehmen, in denen Betriebs- oder Personalräte gewählt sind, aufgrund von einvernehmlichen Vereinbarungen zwischen Arbeitgeber und Betriebs- oder Personalrat, also in einer sogenannten Betriebs- oder Dienstvereinbarung, von diesen festen Vorgaben teilweise abgewichen werden, falls ein Tarifvertrag in dieser Branche so etwas grundsätzlich zulässt, also eine sogenannte „Öffnungsklausel“ enthält (§ 7 ArbZG).

Im Geltungsbereich solcher Tarifverträge ist es auch möglich, wenn kein Personal- oder Betriebsrat besteht, einzelvertragliche Absprachen mit den Mitarbeitern zu treffen, in denen dann ebenfalls Ausnahmen zugelassen werden können ( $\$ 7$ Abs. 3 ArbZG).

Häufig trifft man etwa auf Verkürzung von Ruhezeiten, wenn beispielsweise in der Arbeitszeit ein nicht unerheblicher Anteil Arbeitsbereitschaft oder Bereitschaftsdienste enthalten sind. In solchen Fällen kann auch die Verlängerung der Arbeitszeit vereinbart werden. 


\section{Überstunden und Mehrarbeit}

Eine klar definierte Unterscheidung zwischen Überstunden und Mehrarbeit gibt es häufig nicht, sodass diese Begriffe regelmäßig als Synonym verwendet werden. Etwas anderes gilt für solche Arbeitsverhältnisse, die dem TVöD, also dem Tarifvertrag für den Öffentlichen Dienst, unterliegen. Dieser definiert diese Begriffe ausdrücklich in $\S 6$ TVöD.

Dort, wo keine Betriebsvereinbarung und kein Tarifvertrag den Umgang mit Überstunden festlegen, kommt es auf die einzelvertragliche Vereinbarung zwischen Arbeitgeber und Arbeitnehmer an. Pauschale Bestimmungen, wonach Überstunden mit dem Gehalt abgegolten sind, werden seitens der Arbeitsgerichte häufig als unwirksam betrachtet.

Die meisten Tarifverträge, wie etwa der TVöD oder auch viele Betriebsvereinbarungen, regeln indes, wie mit Überstunden umzugehen ist oder in welchem Zeitraum sie vorher angekündigt werden müssen. Auch die aus Arbeitnehmersicht wichtigste Frage wird darin geklärt: Wie sind die Überstunden zu vergüten? Allerdings sind viele tarifliche Regelungen derart komplex formuliert, dass es ohne die Hilfe eines Anwalts oft nicht geht.

Mit einem vielbeachteten Urteil vom 25. April 2013 hat sich das Bundesarbeitsgericht (6 AZR 800/11) zum Beispiel mit der Frage befasst, ob und wie bei Jahresdienstplänen im Anwendungsbereich des TVöD Überstunden entstehen können. Dazu hat es festgestellt, dass Überstunden bei einer Jahresplanung grundsätzlich nur dann entstehen, wenn über das gesamte Jahr mehr gearbeitet wird, also eine Überschreitung der Jahresarbeitszeit eines vollzeitbeschäftigten Mitarbeiters auftritt.

Es kommt damit grundsätzlich nicht auf die geleisteten Stunden im jeweiligen Schichtrhythmus (hier 6/6-Rhythmus) an, sondern auf die Stunden im gesamten Jahr. Hier bedarf es für die Dienstplangestaltung genauer Kenntnis dieser Rechtsprechung. Allerdings können sogenannte ungeplante Überstunden auch bei einer Jahresplanung zu einer Zuschlagspflicht führen. Hier gilt es, regelmäßig zwischen Vollzeit- und Teilzeitkräften zu unterscheiden.

\section{Wege- und Umkleidezeiten}

Regelmäßig kommt es zu Diskussionen darüber, ob das Umziehen und der Weg innerhalb des Firmengeländes zum tatsächlichen Einsatzort bereits Arbeitszeit ist. Wer morgens im Anzug ins Büro geht, dem stellt sich diese Frage nicht. Die übliche Arbeitszeit beginnt mit Erreichen des Arbeitsorts.

Anders ist es aber da, wo besondere Dienstkleidung vor Arbeitsbeginn angelegt werden muss und dies nicht schon zu Hause geschehen darf. Solche Regelungen finden sich vor allem in Krankenhäusern und Pflegeeinrichtungen, etwa bei OP-Personal und Krankenpflegepersonal, wo insbesondere hygienische Gründe ein Umziehen bereits zu Hause untersagen. Hinzu kommt in größeren Betrieben regelmäßig noch der Weg zwischen Umkleideräumen, etwa im Keller, und dem tatsächlichen Arbeitsort, etwa dem OP in der fünften Etage.

Das Bundesarbeitsgericht hat am 19. September 2012 (5 AZR 678/11) dazu geurteilt, dass die „unter Ausschöpfung der persönlichen Leistungsfähigkeit [...] erforderlichen Umkleidezeiten [...] einschließlich der innerbetrieblichen Wegezeiten von der Umklei- destelle bis zum“ jeweiligen Arbeitsbereich „vergütungspflichtige Arbeitszeit sind“.

Eine pauschale Abgeltung dieser Zeiten soll grundsätzlich durch Betriebs- oder Dienstvereinbarung möglich sein, zumindest im Anwendungsbereich des TVöD. Losgelöst von einer rechtlichen Beurteilung kann schließlich auch der räumlichen Gestaltung, also wo sich die Umkleideräume befinden, eine nicht unbedeutende Rolle zukommen.

\section{Nachtarbeit}

Ein weiteres Thema im Bereich Arbeitszeit ist die Frage, ob Mitarbeiter von ihrer grundsätzlichen Verpflichtung, Nachtdienste zu leisten, befreit werden müssen und nur noch für Tagschichten eingesetzt werden können, wenn dies aus gesundheitlichen Gründen erforderlich ist - oder ob dann eine vollständige Arbeitsunfähigkeit vorliegt.

Der Ausgangsfall, über den das Bundesarbeitsgericht zu entscheiden hatte (BAG vom 09. April 2014 - 10 AZR 637/13), betraf eine Krankenschwester, die aufgrund von zwingender Medikamenteneinnahme keine Nachtdienste mehr absolvieren konnte, da die Gefahr bestand, dass sie aufgrund ihrer Medikation einschlafen könnte.

Der Arbeitgeber, ein großes Krankenhaus, vertrat die Ansicht, dass bei der Krankenschwester eine Arbeitsunfähigkeit eingetreten sei, da auch die Ableistung von Nachtdiensten ihre arbeitsvertragliche Pflicht darstelle, die sie nun unstreitig nicht mehr erbringen könne.

Das Bundesarbeitsgericht sah in der „teilweisen Arbeitsunfähigkeit“" keinen Grund, die Krankenschwester nicht lediglich von den Nachtdiensten auszunehmen und bloß noch für Tagschichten einzuteilen. Diese kann sie weiterhin leisten und damit ihre Arbeitsleistung für den Arbeitgeber erbringen. Andere Arbeitnehmer müssten dann ihre Schichten übernehmen. Eine solche Umorganisation dürfte in den meisten Fällen auch möglich sein.

Schließlich sieht das Arbeitszeitgesetz in $\S 6$ Abs. 4 selbst die Möglichkeit vor, dass Arbeitnehmer von Nacht- und Schichtarbeit bei gesundheitlichen Beschwerden zu befreien sind. Insoweit dürfte es Arbeitgebern ohnehin nahezu unmöglich sein, wegen fehlender Nachtdiensttauglichkeit ein Arbeitsverhältnis zu beenden.

\section{Pausen}

Auch die Pausen gehören zur Arbeitszeit. § 4 des Arbeitszeitgesetzes sieht zwingende Ruhepausen vor. Die Regelung besagt, dass die Arbeit durch im Voraus feststehende Pausen von mindestens 30 Minuten bei einer Arbeitszeit von mehr als sechs bis zu neun Stunden und 45 Minuten bei einer Arbeitszeit von mehr als neun Stunden insgesamt zu unterbrechen ist.

Wer diese Bestimmungen beharrlich ignoriert, den kann die zuständige Aufsichtsbehörde durch einen Verwaltungsakt verpflichten, die Pausenzeiten einzuhalten und die tatsächlich gewährten Pausenzeiten in geeigneter Form aufzuzeichnen und diese beispielsweise monatlich - unbefristet - der Behörde vorzulegen. Dies ist regelmäßig mit einem immensen Verwaltungsaufwand und entsprechenden Kosten verbunden.

Darüber hinaus können Bußgelder bis zu 15000 Euro allein schon bei Fahrlässigkeit verhängt werden. Bei Vorsatz oder be- 
harrlicher Wiederholung droht sogar eine Freiheitsstrafe für den Inhaber der Unternehmung. Aus diesem Grund sollte auch der Pause entsprechende Aufmerksamkeit gewidmet werden.

\section{Rufbereitschaft und Bereitschaftsdienst}

Nach langjährigen gerichtlichen Auseinandersetzungen steht seit der grundlegenden Simap-Entscheidung des Europäischen Gerichtshofs aus dem Jahre 2000 fest, dass Bereitschaftsdienst als Arbeitszeit zu werten ist. Der Gesetzgeber hat dies in § 7 Abs. 1 a ArbZG im Jahre 2004 schließlich auch im Arbeitszeitgesetz verankert.

Beim Bereitschaftsdienst ist der Arbeitnehmer verpflichtet, sich an einem vom Arbeitgeber bestimmten Ort aufzuhalten, um jederzeit die Arbeit aufnehmen zu können. Davon losgelöst bleibt aber weiterhin die Frage, ob und in welcher Höhe Zeiten des Bereitschaftsdienstes zu vergüten sind. Viele Tarifverträge regeln die Vergütung für Bereitschaftsdienst abweichend von der üblichen Bezahlung. Häufig gibt es pauschale Zahlungen, so etwa im TVöD. Ist tariflich nichts vereinbart, ist grundsätzlich die normale Bezahlung zu leisten.

Anders sieht es bei der Rufbereitschaft aus. Hier kann der Arbeitnehmer frei wählen, wo er sich aufhält, er muss nur erreichbar und dann in der Lage sein, innerhalb einer angemessenen Zeitspanne (regelmäßig 30 bis 60 Minuten) an seiner Arbeitsstätte einzutreffen und seine vertragliche Arbeit aufzunehmen. Rufbereitschaft zählt ausdrücklich nicht zur Arbeitszeit.

Erst wenn der Arbeitnehmer tatsächlich zur Arbeit gerufen wird, läuft ab der „Heranziehung“ zur Arbeit die „Stechuhr“ und er ist normal zu vergüten. Dabei gilt bereits der Weg zur Arbeitsstätte als vergütungspflichtige Arbeitszeit. Wird der Arbeitnehmer während der Rufbereitschaft nicht zur Arbeitsleistung herangezogen, so erwirbt er auch keinen normalen Vergütungsanspruch, sondern erhält regelmäßig nur eine deutlich geringere, meist tariflich festgelegte Pauschale.

\section{Stand-by-Dienste}

Diese beiden wesentlichen Unterschiede zwischen Bereitschaftsdienst und Rufbereitschaft regen die Kreativität manch Berater an. Das Ziel lautet: möglichst viel Flexibilität über die Rufbereitschaft der Mitarbeiter zu generieren. Denn diese ist, wie gezeigt, weder Arbeitszeit noch wie solche zu vergüten. Bereitschaftsdienst hingegen ist in der Regel deutlich höher vergütet und zählt zur Arbeitszeit im Sinne des Arbeitszeitgesetzes, auch wenn keine Arbeitsleistung anfällt.

Vor diesem Hintergrund werden aktuell landauf, landab die sogenannten „Stand-by-Modelle“ oder „Flexi-Modelle“ als Lösung aller Sorgen präsentiert. Die Idee dahinter ist recht einfach: Es wird einfach kein Bereitschaftsdienst oder keine Rufbereitschaft mehr angeordnet. Mitarbeiter haben sich vielmehr innerhalb bestimmter Zeitfenster erreichbar zu zeigen. Fällt ein Mitarbeiter in einer Schicht aus, so kann der Mitarbeiter im „Stand-by“ während dieser Zeitfenster angerufen und zum entsprechenden Dienst herangezogen werden. Wird er nicht angerufen, weiß er, dass er „frei“ hat. Also alles gut?

Das vermeintlich für alle gerechte und faire Dienstplan-Gestaltungstool erweist sich bei genauerer Betrachtung als bloße Schi- märe. Ein „Einspringen im Frei“ kann vom Arbeitgeber grundsätzlich nicht kurzfristig angeordnet werden; dienstplanfrei bedeutet grundsätzlich auch dienstplanfrei.

Das Problem ist vielmehr häufig hausgemacht. Die Personalengpässe entstehen schon bei der Berechnung und Festlegung des notwendigen Personalbedarfs. Wird hier bereits aus Kostengründen „auf Kante genäht“, zieht sich dieser Mangel wie ein roter Faden durch die sich anschließende Dienstplangestaltung und die gelebten Dienste.

„Einspringen im Frei“ gehört deswegen in vielen Häusern und Einrichtungen zum Tagesgeschäft, auch wenn es regelmäßig nicht mit dem geltenden Arbeitsrecht im Einklang steht.

Zudem erweist sich das neue Modell der Stand-by-Dienste nur als alter Wein in neuen Schläuchen. Bei genauerer Betrachtung zeigt sich, dass es nichts anderes ist als Rufbereitschaft. Der Mitarbeiter im Stand-by gewinnt weder Freizeit noch Flexibilität. Sein Tag ist eben nicht frei planbar und der Mehrwert an Flexibilität für den Arbeitnehmer hat erhebliche Grenzen. Wird er zudem noch um 14 Uhr zum Nachtdienst gerufen, ist der Tag seit 5.30 Uhr voll verplant, aber erst ab 14 Uhr angemessen vergütet.

Schließlich gibt es auch massive rechtliche Bedenken, regelmäßige „Dienstplanausfälle“ über eine „atypische“ Rufbereitschaft kompensieren zu wollen. Wie hat es ein Teilnehmer bei einer Veranstaltung zum Arbeitszeitrecht treffend ausgedrückt: „Stand-byDienste [...] ist doch neumodischer Käse!“. Diesem Urteil können sich die Autoren auch aus juristischer Sicht nur anschließen.

\section{Workshop}

Sie wollen zu „Stand-by und Co“ mehr wissen? Dann buchen Sie den Tagesworkshop „Arbeitszeit und Dienstplangestaltung“ des Bundesverbands Pflegemanagement.

\section{E AUTOREN}

Daniel Balzert, LL. M.

Rechtsanwalt, Fachanwalt für Arbeitsrecht, Master of Law \& Economics

Dr. Tobias Weimer, M. A.

Rechtsanwalt, Fachanwalt für Medizinrecht, Master of Arts - Management von Gesundheitseinrichtungen WEIMER | BORK - Kanzlei für Medizin-, Arbeits- $\mathcal{E}$ Strafrecht Frielinghausstr. 8, 44803 Bochum www.kanzlei-weimer-bork.de info@kanzlei-weimer-bork.de
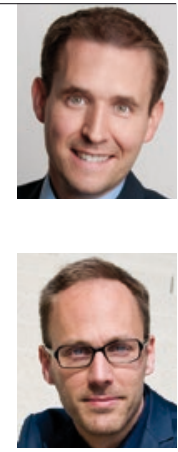

B BIBLIOGRAFIE

DOI 10.1055/s-0041-109830

Im OP 2016; 2: 54-56

(c) Georg Thieme Verlag KG

Stuttgart · New York · ISSN 1611-7905 